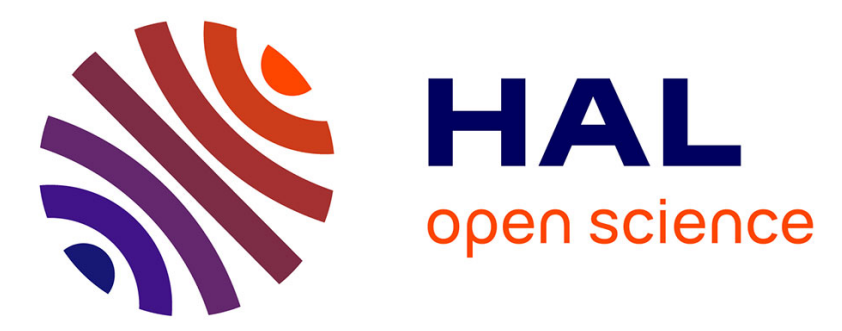

\title{
A Hidden Power of Ontology Charts from Affordances to Environmental States
}

\author{
José Cordeiro
}

\section{To cite this version:}

José Cordeiro. A Hidden Power of Ontology Charts from Affordances to Environmental States. 18th International Conference on Informatics and Semiotics in Organisations (ICISO), Jul 2018, Reading, United Kingdom. pp.105-114, 10.1007/978-3-319-94541-5_11 . hal-01920746

\section{HAL Id: hal-01920746 \\ https://hal.inria.fr/hal-01920746}

Submitted on 13 Nov 2018

HAL is a multi-disciplinary open access archive for the deposit and dissemination of scientific research documents, whether they are published or not. The documents may come from teaching and research institutions in France or abroad, or from public or private research centers.
L'archive ouverte pluridisciplinaire HAL, est destinée au dépôt et à la diffusion de documents scientifiques de niveau recherche, publiés ou non, émanant des établissements d'enseignement et de recherche français ou étrangers, des laboratoires publics ou privés. 


\title{
A Hidden Power of Ontology Charts from Affordances to Environmental States
}

\author{
José Cordeiro \\ Setúbal School of Technology, Polytechnic Institute of Setúbal, Campus do IPS, Estefanilha, \\ 2914-508 Setúbal, Portugal \\ jose.cordeirodestsetubal.ips.pt
}

\begin{abstract}
Ontology Charts are a powerful model element in Organisational Semiotics used to depict a stable and precise view of a business system. They show affordances and ontological dependencies as, respectively, nodes and links between them. Spite their apparent simplicity, they are many times hard to create. In this paper we refer to some common issues regarding the creation of Ontology Charts. Nevertheless, they have a hidden power of defining anchors for a stable information system supported by the affordance concept. In NOMIS - a NOrmative Modelling of Information Systems - affordances are transformed to states and environmental states and used as stable state paths within an information system. This paper shows how this transformation takes place and some advantages and possibilities of using it.
\end{abstract}

Keywords: Information Systems, Information Systems Modelling, HumanCentred Information Systems, Organisational Semiotics, Ontology Charts, NOMIS, NOMIS Views, States, Environmental States, Business Processes, Goal and Context Modelling.

\section{Introduction}

Organisational Semiotics (OS), as proposed by Stamper [1], [2] uses Ontology Charts as a diagrammatic language in which an organisation or business is modelled. In these diagrams, two types of elements are represented: affordances and ontological dependencies. These are key concepts in OS. A main use of Ontology Charts (OC) is in the elicitation of information system requirements. OCs offer a stable and precise view of organisational requirements. Spite their simple appearance, they are hard to produce with required accuracy. On the other hand, NOMIS, a modelling approach to information systems proposed in [3], uses other diagrams, similar to OCs, where a new modelling element known as Environmental State (ES) is used. This is an adaptation of the affordance concept. Also ontological dependencies are adapted to existential dependencies in NOMIS. This adaptation reveals the power of Ontology Charts. 
In this paper, we make a brief description and analysis of Ontology Charts and we show how OS affordances and ontological dependencies became environmental states and existential dependencies, respectively, in NOMIS. Moreover, we emphasise the advantages and power of using ES and its associated diagrams and we point some research directions to explore these concepts.

This paper is organised as follows: section 2 gives a brief overview and analysis of Ontology Charts, section 3 presents NOMIS and its State View, referring to Environmental States, Existential dependencies and its correspondence to affordances and ontological dependencies, section 4 emphasises the power of Environmental States, providing some ideas for further research, section 5 describes some related work and section 6 concludes, pointing future work.

\section{Ontology Charts}

Ontology Charts (OC) are used in Organisational Semiotics to represent graphically a business map of an organisation, its ontological schema. In these charts, we find affordances and ontological dependencies as, respectively, nodes and lines connecting them. Affordances, are the most important element in OCs representing the organisation key business terms. By using these charts, meaning of any business term is made clear and precise. OCs are the most stable representation of an organisation. In Organisational Semiotics, OCs are an artefact, created by the MEASURE methods ([4]) for requirements elicitation. In particular, OCs are the outcome of applying the Semantic Analysis Method (SAM) to an organisational problem. In the next sections, we detail some simple aspects of OCs relevant to this work, the way they are produced and we make an overall analysis.

\subsection{Agents and Affordances}

According to Gibson's theory of perception [5], adapted by OS, affordances are the invariants which the agent recognises from the flux of information he/she perceives from the environment. In OS, each affordance represents a state of affairs affording or making possible for the agent some repertoire of behaviour. An affordance can be simply described by "what the environment can do for an agent" or "what it affords an agent to do". As an example from [6], an agent with a pen (an invariant) affords him/her the capability of writing. Also, an agent with a pen and a piece of paper will be able to write a letter. A pen affordance for a certain human agent will assign him the ability to perform a set of actions such as writing. Affordances, shown as nodes, are represented as words in OCs that are selected from the business domain description as the supporting elements of its organisational behaviour. In Error! Reference source not found., there is an example of an OC of a conference organisation. In this OC we observe, as nodes, key terms from this activity, such as participant, work, program committee, conference, etc. 


\subsection{Ontological Dependency}

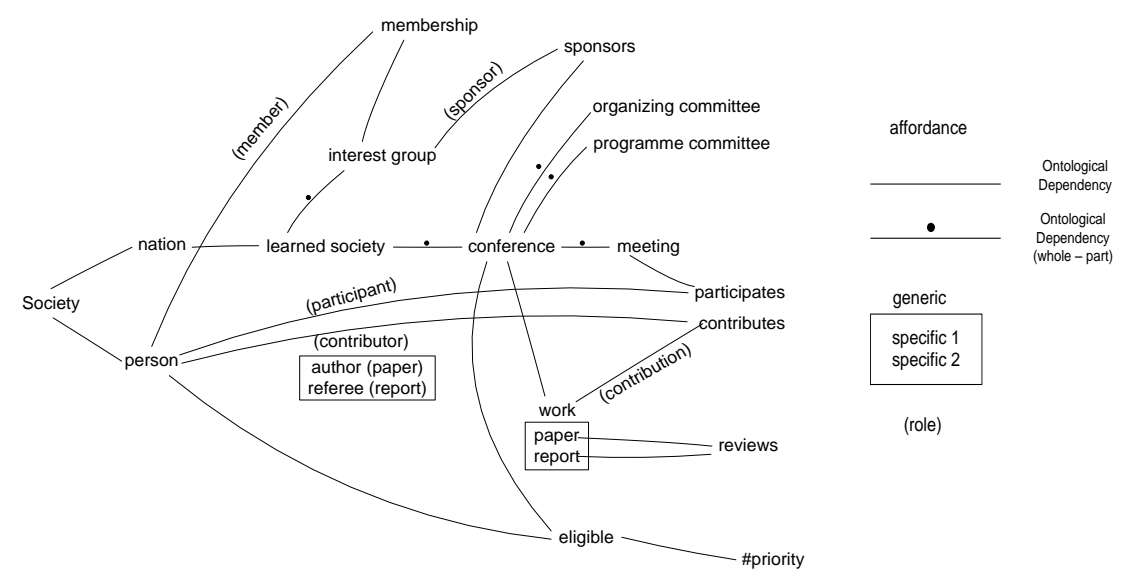

Fig. 1. Ontology Chart of a conference [1].

Ontological dependencies (OD) are relationships between affordances where one affordance - the dependent - cannot exist without the co-existence of another one - its antecedent. Behaviour afforded by a dependent affordance can only be realised when the behaviour of its antecedent is being realised [1]. While affordances are represented as words in OCs, ODs are represented as solid lines (arcs) connecting these words. Also, in OCs antecedent affordances are shown on left side and dependent affordances on the right side making OC a directed chart. As an example of an OD, there is no conference sponsor without the coexistence of an interest group as sponsor and a conference, this is shown in Error! Reference source not found. using two arcs connecting these affordances. An important effect of ODs is that, if an antecedent affordance ceases to exist, then all dependent affordances ends their existence as well. For example, without a conference, the meeting, the person as a participant and his/her work as a contribution stop all their existence in this context. There are also two rules to follow when creating OCs related to ODs. First, the most left affordance is always the society, a root node from where all other affordances depend directly or indirectly. Without society, any organisation or business, would not have any meaning. The remaining rule is that an affordance cannot have an OD from more than two other affordances. In this case, a simple justification is that it is not needed.

\subsection{Semantic Analysis Method}

As mentioned before, the Semantic Analysis Method of MEASURE has, as its outcome, an Ontology Chart. This method starts from a problem specification and 
produces a conceptual schema or 'business map' of an organisation (an OC). According to [2], conducting a Semantic Analysis involves four steps:

1. Problem definition - To understand the problem domain and collect the terms to be used in the semantic analysis.

2. Candidate affordance generation - Each identified term or semantic unit will be selected as a candidate affordance for the next step.

3. Candidate grouping - Selected affordances will be categorised as affordances or agents. Next, prior to the complete OC, identified elements will be used to create small chunks of interconnected affordances related by ontological dependencies.

4. Ontology charting - Connects all chunks of affordances and pieces in a unique Ontology Chart that represent the final ontological schema of the business system.

The business terms or affordances in OCs are usually identified and established by business stakeholders contributing also to their preciseness.

\subsection{Analysis of Ontology Charts}

In the creation process of OCs, is relatively easy to identify affordances because anything in the problem description can be an affordance. In fact, an affordance is something in the environment seen as "an invariant within the flux of information" that affords an agent some action. It can be a reference for a physical object, an agent, an organisation term, etc. Usually affordances are enablers of actions but do not represent themselves actions, therefore verbs are rarely used as affordances. In OS theory, Stamper added the notion of a social affordance where some social concepts can also act as affordances, for example, contracts, organisations, society, nation, etc. Additionally, there is a distinction between regular affordances and agent affordances, where agents are human actors or organisations. Therefore, although easy to find they may be difficult to select. There are no precise rules or guidelines for this selection. A second difficulty in the recognition of affordances is that some of them are not included in the problem description, usually are tacit knowledge, such as learned society or nation in Error! Reference source not found.. This leads to another consequence: getting affordances from a general domain may lead us to add other affordances that are essential but not important for the problem description resulting, possibly, in an over specification problem. Another difficulty is to link the different affordances using ODs in chunks of connected affordances. In general, an OD is a dependency of a kind of state on another state where these states are represented as affordances. The first state is a permanent state that must exist whenever the second state exists. If this first states ends, the second state and all connected states end their existence as well. This means that a transient state should not be taken as an affordance and, particularly, that mutually exclusive states should not depend on one another. As an example, consider an open and closed door situation. We may say that an open door is an affordance allowing a close action. Also, we only have a closed door affordance if the door is previously open (we cannot close a closed door). We may be tempted to create an OD between the open and the closed door but this is wrong as they behave as transient states. A last problem, 
related to ODs is the dependency nature, which varies according to a specific view. Looking to Error! Reference source not found., is possible to identify different hidden types of ODs. The existential dependency between participant and person is, clearly, a physical dependency. On the other hand, the OD between sponsor and conference should be understood as a legal dependency. Other views can be identified confusing an inexperienced OC creator.

Nevertheless, an OC is in fact rather unique among the different Information Systems methodologies. None of them take into account important 'existential' dependencies between their elements. Also, a "correct" OC may lead to a very stable representation of a business organisation, making it to survive to requirement changes.

\section{NOMIS State View Representation}

NOMIS, described in [3], is a human centred information systems modelling approach based on human observable actions. It proposes a vision of information systems composed by a set of views addressing human interaction, action processes and context for actions inspired and based on, respectively, Enterprise Ontology (EO) [7], the Theory of Organized Activity (TOA) [8], and Organisational Semiotics (OS) [2]. In this section we describe the NOMIS state view, based on OS, and we will describe its relation to Ontology Charts.

\subsection{NOMIS brief Overview}

NOMIS is a social-technical approach that understands information systems "as human activity (social) systems which may or may not involve the use of computer systems" [9]. It is based on human observable actions, those perceived by the human sensory system. This focus is originated by its foundational philosophical stance - Human Relativism [10] - that sees observable reality as "more consensual, precise and, therefore more appropriate to be used by scientific methods".

NOMIS proposes a vision of an information system composed by four complementary views - Interaction View, Physical View, State View and Information View - addressing, respectively, human interactions, action processes, context for actions, and information. For modelling purposes, it provides a set of diagrams a tables utilized by each of these views. The elements represented in NOMIS views correspond to key concepts in NOMIS, namely:

- Human observable actions

- Actors - human performers

- Bodies - physical things

- Information Items - information without any physical support

- Language Actions (or Coordination-acts)

- States and Environmental States

In this paper we focus on the State View, inspired by OS, in particular its Ontology Charts, which is described in next section. 


\subsection{NOMIS State View}

NOMIS state view looks into environmental conditions or states that enable a human agent to act. This is an adaptation of the OS affordance concept. In this case, it distinguishes two types of states:

1. A simple state - only applied to physical things (bodies). If applied to an agent takes the form of a role.

2. An environmental state - a composite of NOMIS elements, namely, bodies and actors, possibly in a specific state, and actions. It is used to represent essential business states.

States, in these view, may be related to other states by existential dependencies. This is also an adaptation of Ontological Dependencies. Furthermore, there are three different types of diagrams used to represent this view:

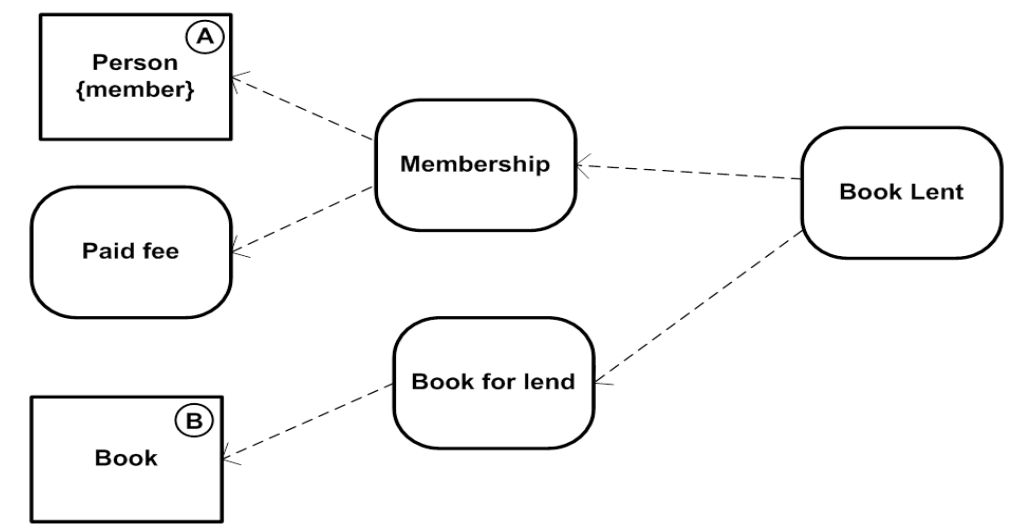

Fig. 2. An Existential Dependency Diagram of a library system [3]

1. Body State Diagram (BSD) - shows the different states of a single body.

2. Existential Dependencies Diagram (EDD) - shows Environmental States and their existential dependencies. This is similar to Ontology Charts.

3. Environmental State Diagram (ESD) - details each Environmental State by showing its composing elements.

In Error! Reference source not found., there is an example of an EDD of a library system. It results from modelling a simple use case of a library system described in [7]. In this figure, we can see Environmental States (ES) represented by round corner rectangles. A Book Lent ES depends on the existence of an ES of Book in a for lend state and a valid membership state.

\subsection{Environmental States and Affordances}

Environmental States and their existential dependencies, in NOMIS, are inspired by OS affordances and their ontological dependencies but they differ in many aspects. An ES 
is a collection of one or more elements precisely defined. In this case, an ES is reached if each of its composing elements is present. Because NOMIS models Information Systems and intends, mainly, to support development and implementation of computerised information systems, each NOMIS element is, in fact, information inside a computer. So, physical things (bodies), persons, actions, and everything else is just stored data. Looking at Error! Reference source not found.Error! Reference source not found., a membership ES depends on a person, registered in the system, as a library member, and his/her paid fee information. If the contact information of this person is lost or if she/he did not pay her/his membership fee, the ES state is not valid. Also, the existential relationship between ES applies: a book is not in a lent state if there is no member lending it. In NOMIS, ES are always related to the data where they are based and must be though as it.

Existential Dependencies, are also different from Ontological Dependencies. Existential Dependencies relates Environmental States and are always data dependency states, contrary to ontological dependencies that can exhibit dependencies according to some specific view, for example, physical or legal views.

For representation purposes, EDD can be used to reproduce Ontology Charts but they do not have to follow its rules. There is no society or any other root node, neither is necessary to have a limitation of a maximum of two dependents in each dependency.

\section{The Environmental State Advantage}

Environmental states, inspired by OS affordances, are expected to exhibit a similar power in modelling an organisation in a precise and stable way. Besides their representational benefits, they are intending to have a practical application in information systems development. In this section, we address some possible advantages of using Environmental States and trace a research path to explore this new way of looking and representing information and business systems. 


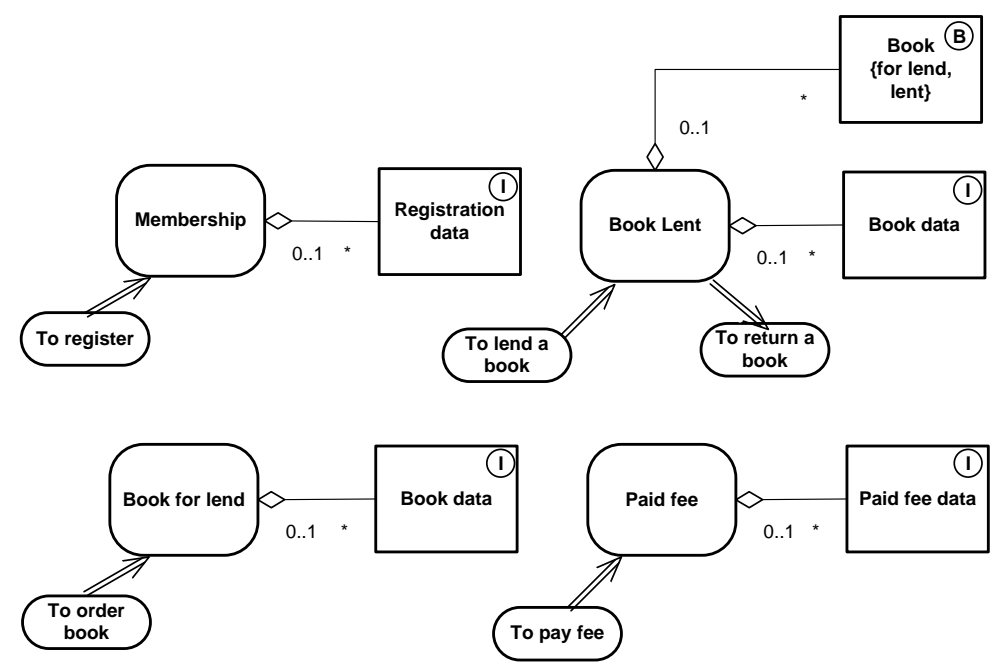

Fig. 3. An ESD of a library system [3].

\subsection{Environmental State Modelling}

As mentioned before, EDD diagrams, such as the one shown in Error! Reference source not found., shows Environmental States, its composing elements, and its existential dependencies. A more detailed diagram is the ESD diagram that adds to EDDs a representation of actions or activities entering or leaving a state. In ESDs, entering actions or activities are represented in a round corner rectangle with leaving arrows pointing to an ES. This action correspond to a necessary process to reach that state. For example, in Error! Reference source not found. representing an ESD of a library system, there is a lend book activity before reaching the lent book ES. This activity could be implemented as a typical business process of the library system. On the other hand, leaving actions or activities, are in fact actions enabled by that state. Similar to affordances, allowing for certain actions, these actions are also allowed by the correspondent ES. It is the case of return a book action that is available from a book lent ES. What is impressive in the ESD shown in Error! Reference source not found. is that it shows a complete overview of the library system, including the different business process identified in this case study. Additionally, the ES states represented should be considered stable. It is possible to change, for example, the register, order book, lend book business processes and the ESD is still valid. Moreover, these states can be seen as information system anchors for a specific system, supporting different action or activities. A research question is: can we model other systems with ES diagrams, such as they represent an overall stable and usable view of that system? 


\subsection{Environmental States and Business Processes}

NOMIS is based on human observable actions. Therefore, any action or activity is always related to a human performer. A business process represented as a sequence of actions, in NOMIS, involves exclusively human actions. As a consequence, a particular sequence of actions may not be followed by a human performer, these actions are not hard coded in a system. Anyway, ESs represent a composite of elements that may have their related data stored in a system. We may use this data to support any (human) business process. In this case, ESs are the support for business processes. Also, they are the system stable parts, allowing business processes to change and adapt to the context. This could be a major advantage, as business processes are usually dynamic, changing and making information system to change often. Also, another research question, is to assess the system adaptability and responsiveness for business processes changes.

\subsection{Environmental States and Context/Goal Modelling}

Environmental States exhibit another interesting properties. They can act as context or as goals. Regarding context, an ES is a state (or a kind of affordance) that allows for some actions or activities. This can be seen and modelled as context. Effectively, it is possible to represent context as an ES. In the library system case study, the lend a book action is possible during library open hours, in a specific library desk. The desk and the timetable may be modelled as ESs elements required for that action.

Concerning goals, ESs can also be understood as goals. Taking as example the same case study, in this library system a goal is to have books for lend and registered members to lend those books. Both goals relates to system states: books in a for lend state and registered members in a membership. This is a perfect match with the ESs identified before. So, a last research question is: can we map goals and context modelling to ESs models, and provide a precise and applicable base for them?

\section{$5 \quad$ Related Research}

Ontology Charts are analysed in [11] where they are compared to a few other modelling techniques. There are a few attempts to use OCs in formal ways within information system development. Firstly, in [12], some heuristic rules for UML class diagram derivation from OCs are proposed. In this work are just given some simple hints on how to obtain (and translate) the OC elements into UML elements. These UML elements are limited to classes and associations, compositions and generalizations relationships among then. In [13], Ontology Charts are modelled with UML profiles. In this work, UML adaptation issues related to Ontology Charts, are reported. [14], proposes the generation of a prototype system from Ontology Charts. The solution uses a database structure to store information from the elements in the OC. [15] suggested later the generation of UML 2 use cases from Ontology Charts. They map agents to actors and communication acts to use cases. This transformation is not suitable as well for NOMIS as it does not cover the required detailing. Also, in NOMIS there is a current 
proposal in [16] to use a Model-Driven System Development approach to derive application code from NOMIS Models. In this case, EDD are supposed to be implemented as state machines, inside a specific business middleware.

\section{Conclusions and Future Work}

In this paper, Ontology Charts are presented together with its key concepts of affordances and ontological dependencies. This artefact, used in Organisational Semiotics to represent key business terms, is analysed and some difficulties in creating OCs are reported. On the other hand, an equivalent diagrammatic representation, the Environmental Dependency Diagram, used by NOMIS is introduced. This EDD is used by NOMIS State View to represent environmental states and its existential dependencies. EDDs were inspired by OC, and also, its elements are versions of affordances and ontological dependencies. The relation between both diagrams is explained and the EDD power, inherited by OCs is revealed. Some advantages an research directions are pointed for EDDs.

As future work, EDDs and other modelling diagrams used in NOMIS, are planned to be used by a Model-Driven System Development approach. In this case, a new domain specific language is to be created for expressing NOMIS, and some model transformations will be created and applied to derive part of an information system application code as reported in [16].

\section{References}

1. Stamper, R. Signs, Norms, and Information Systems. In Signs of Work, Holmqvist B. et al (eds). Walter de Gruyter, Berlin (1996).

2. Liu, K. Semiotics in Information Systems Engineering. Cambridge University Press, Cambridge, UK (2000).

3. Cordeiro, J. Normative Approach to Information systems Modelling. PhD Thesis. The University of Reading, UK (2011).

4. Stamper, R., Althans, K., and Backhouse, J. MEASUR: Method For Eliciting, Analysing and Specifying User Requirements. In Computer Assistance During the System Life Cycle, Olle, T. W., Verrijn-Stuart, A.A. and Bhabuta, L. (eds), Proceedings of the IFIP WG 8.1 Working Conference on Computerized Assistance During the Information Systems Life Cycle, CRIS 88, Egham, England, 19-22 September, 1988. North Holland (1988) 67-115.

5. Gibson, J. The Ecological Approach to Visual Perception. Houghton Mifflin, Boston (1979).

6. Stamper, R. New Directions for Systems analysis and Design. In Enterprise Information Systems, Joaquim Filipe (ed). Kluwer Academic Publishers, Dordrecht, The Netherlands (2000).

7. Dietz, J. Enterprise Ontology, Theory and Methodology. Springer-Verlag, Berlin Heidelberg, Germany (2006).

8. Holt, A. Organized Activity and Its Support by Computer. Kluwer Academic Publishers, Dordrecht, The Netherlands (1997). 
9. Buckingham, R. A., Hirschheim, R. A., Land, F. F., and Tully, C. J. (eds). Information systems curriculum: A basis for course design, Information Systems Education: Recommendations and Implementation. Cambridge University Press, Cambridge (1987).

10. Cordeiro, J., Filipe, J. and Liu, K. Towards a Human Oriented Approach to Information Systems Development. In Proceedings of the 3rd International Workshop on Enterprise Systems and Technology. Sofia, Bulgaria (2009).

11. Cordeiro, J. and Filipe, J. Comparative Analysis of Ontology Charting with other Modelling Techniques. In Proceedings of the 8th International Workshop on Organisational Semiotics. Toulouse (2005).

12. Bonacin, R., Baranauskas, M. C. C. and Liu, K. From Ontology Charts to Class Diagrams Semantic Analysis Aiding Systems Design. In Proceedings of the 6th International Conference on Enterprise Information Systems, ICEIS 2004, Porto, Portugal. v. 1. (2004) 389-395.

13. Cordeiro, J. and Liu, K. UML 2 Profiles for Ontology Charts and Diplans - Issues on Metamodelling. In Proceedings of the 2nd International Workshop on Enterprise Modelling and Information Systems Architectures. St. Goar, Germany (2007).

14. Tsaramirsis, G. and Poernomo, I. Prototype Generation from Ontology Charts. In Fifth International Conference on Information Technology. New Generations, Las Vegas, NV, (2008) 1177-1178.

15. Tsaramirsis, G. and Yamin, M. Generation of UML2 Use Cases from MEASUR's Ontology Charts: A MDA Approach. In Model-Driven Business Process Engineering, eds. Lano, K., Zandu, R. and Maroukian, K. page 67 - 76. ISBN: 978-1-60805-893-8. Shariqah, United Arab Emirates, Bentham Science Publishers Ltd (2014).

16. Cordeiro, J. Developing Information Systems with NOMIS - A Model-Driven System Development Approach. In Proceedings of the Seventh International Symposium on Business Modeling and Software Design. Barcelona, Spain (2017). 